\title{
Regional recurrence in a case series of patients with carcinoma of the nasal cavity - therapeutic implications*
}

\author{
Timon Hussain, Stefan Mattheis, Nina Dominas, Benedikt Höing, \\ Stephan Lang, Boris A. Stuck
}

Department of Otorhinolaryngology, Head and Neck Surgery, Essen University Hospital, University of Duisburg-Essen, Germany

\author{
Rhinology 55: 355-362, 2017 \\ https://doi.org/10.4193/Rhino17.088
}

*Received for publication:

May 1, 2017

Accepted: July 24, 2017

\begin{abstract}
Introduction: Surgery is the primary treatment option for squamous cell carcinomas of the nasal cavity (NCSCC). Nodal involvement is rare at the time of initial diagnosis, and the role of diagnostic neck dissection as well as potential adjuvant irradiation of the neck remains controversial. The objective of this study was to assess the oncologic outcomes of patients with NCSCC with special emphasis on cervical lymph node treatment and recurrence.
\end{abstract}

Methods: 37 previously untreated patients were included in this study. Demographic data, tumor characteristics, therapeutic management, and clinical outcome were analyzed.

Results: Patients with advanced stage tumors were slightly overrepresented in this study and overall 3-year disease-free survival was $63 \%$. A surgical treatment approach was pursued in $89 \%(n=33)$ of patients. After total rhinectomy, there were no cases of local recurrence. Overall, $12 \%(n=4)$ of all surgically treated patients developed local recurrence. While in $45 \%$ of patients ( $n=15)$, neck dissection was performed at the time of tumor resection, initial lymph node metastasis was histologically confirmed in only $6 \%(n=2)$ of surgically treated patients. Cervical lymph node recurrence occurred in $18 \%(n=6)$ of patients, predominantly those with advanced stage tumors, despite prior treatment of the neck.

Conclusion: Excellent local control is achievable for patients with NCSCC, especially with radical tumor resection. While cervical lymph node metastasis is rare at the time of diagnosis, regional lymph node recurrence needs to be taken into consideration when planning therapy and follow-up. Multimodal treatment of the neck may be required for patients with advanced stage tumors.

Key words: nasal cavity carcinoma, lymph node metastasis, rhinectomy, neck dissection

\section{Introduction}

Squamous cell carcinomas of the nasal cavity (NCSCC) are rare malignancies, accounting for only $3 \%$ of head and neck neoplasms ${ }^{(1)}$. The specific tumor site was only added to the American Joint Committee on Cancer (AJCC) staging system in 2003, hereby allowing for standardized analyses. NCSCC include carcinomas of the floor, the lateral walls, the septum and the vestibule. They are currently grouped together with cancers of the ethmoid sinus for staging purposes (Table 1). However, in recent years, studies have begun to address the specific oncologic characteristics and outcomes of NCSCC. It has since been established in large population-based analyses that NCSCC is a disease which is found more commonly in males than in females, and that smoking is an important risk factor ${ }^{(2)}$. Overall incidence rates for NCSCC have been slightly declining over the past decades; however, despite the introduction of improved treatment protocols for head and neck cancer, there has not been an increase in survival ${ }^{(3)}$. This has prompted investigations aiming to better understand the particular oncologic characteristics of this disease and potentially improve treatment strategies.

Since the primary tumor site is easily accessible and even advanced stage tumors can be mostly resected with sufficient surgical 
margins, surgery is accepted as the primary treatment modality for NCSCC. Distant metastases are extremely rare at the time of diagnosis, irrespective of tumor stage. It is important to note in this context, that T-stage is not merely defined by tumor size but rather by involvement of anatomic subsites and the skin. The role of neck dissection and adjuvant treatment of the neck remains somewhat unclear. Undoubtedly, lymph node status is a highly relevant prognostic factor. As shown by Unsal et al. in a population-based analysis, disease specific survival drops from approximately $75 \%$ for patients without nodal involvement to less than $40 \%$ for patients with regional lymph node metastasis ${ }^{(2)}$. At the same time, lymph node involvement at time of diagnosis is rare for NCSCC compared to other head and neck malignancies. It is reported to be around $9 \%$ for all stages, ranging from around $4 \%(\mathrm{~T} 1)$ to $21 \%(\mathrm{~T} 4)^{(2)}$. Hence, in the majority of NCSCC cases, patients will clinically present with an NO neck at the time of diagnosis. In the literature, elective treatment of the NO neck for head and neck cancers is generally supported, when the rate of occult metastases is suspected to be greater than $20 \%{ }^{(4,5)}$. Only late stage NCSCC patients meet these criteria. Importantly though, a recent meta-analysis reported a relatively high regional recurrence rate across all stages of $18.1 \%$, despite the low rate of lymph node metastasis at the time of diagnosis ${ }^{(6)}$. In addition, the study showed that the use of prophylactic irradiation of the neck significantly reduces the risk of regional recurrence. These findings raise the question, whether prophylactic treatment of the neck should be considered for patients with NCSCC, despite presentation with an N0 neck at the time of diagnosis. Currently, no clear treatment guidelines exist, in part due to the rarity of the disease and resulting lack of data. In this study, we therefore assessed oncologic parameters, therapeutic approaches, and outcomes of patients with NCSCC with a focus on the treatment of the neck and regional recurrence. While the numbers of patients included in single-center study cannot match those of population-based analyses, here, individual treatment approaches can be assessed in detail and correlated with oncologic outcomes hereby potentially providing insights, which might assist physicians in the decision-making process and ultimately improve patient survival.

\section{Patients and methods}

Previously untreated patients with histologically confirmed squamous cell carcinoma of the nasal cavity who were newly diagnosed between 2005 and 2016 were included in this study. Patients with carcinomas originating from the paranasal sinuses including the ethmoid, were excluded. Initial diagnosis, staging, and treatment, as well as follow-up for all patients were performed at the same tertiary center, allowing for full access and comparability for all relevant oncologic parameters.

All patients are routinely followed up for five years after initial treatment at regular intervals at the center thus ensuring timely

Table 1. AJCC staging system for tumors of the nasal cavity and ethmoid sinus.

\section{Stage}

$\mathrm{T} 1$

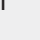

$\mathrm{T} 2$

Invades 2 subsites in a single region or extends to involve an adjacent region within the naso-ethmoidal complex, with or without bony invasion

T3

Extends to invade the medial wall or floor of the orbit, maxillary sinus, palate, or cribriform plate

Invades any of the following: anterior orbital contents,

T4a skin of nose or cheek, minimal extension to anterior cranial fossa, pterygoid plates, sphenoid, or frontal sinuses

Invades any of the following: orbital apex, dura mater,

T4b brain, middle cranial fossa, cranial nerves other than V2, nasopharynx, or clivus

Union for International Cancer Control (UICC) Staging System for Tumors of the Nasal Cavity and Ethmoid Sinus. Adapted from: UICC 8th Edition Cancer Staging Manual.

detection of potential recurrence. Follow-up intervals at the tertiary care center were every 8 weeks during the first year, every 3 months during the second year, every 6 months during the third year and every 12 months for the fourth and fifth year after primary treatment. Importantly, in between every follow-up visit at the center, an additional follow-up examination was performed by the patients' resident otolaryngologist. Every follow-up visit included a thorough clinical exam, as well as an ultrasound scan of the neck. Patients without regular follow-up records were not included in this study. Main reasons for interrupted clinical follow-up within the first five years after initial treatment were non-related severe diseases, and patient incompliance.

Treatment decisions were made in an interdisciplinary tumor board based on tumor stage and location, as well as lymph node status as assessed by sonography and via CT-scan.

Paper-based and electronic patient charts were reviewed to gather patient information, including demographic data, as well as oncologic parameters, such staging results, treatment regimens, and follow-up results.

Kaplan-Meier plotting to represent disease-free survival was performed with SPSS statistics software.

\section{Results}

37 patients who met the inclusion criteria were identified. Mean age was $62.9 \pm 9.0$ years, ranging from 49 to 84 years. Distribution between genders was nearly even, the study included 19 male patients and 18 females.

\section{Tumor characteristics}

Tumor characteristics are summarized in Table 2. In the analyzed 
Table 2. Tumor characteristics of patients included in the study at initial diagnosis.

\begin{tabular}{|l|l|}
\hline Characteristics & No. of patients (\%) \\
\hline T stage & $11(30)$ \\
\hline T1 & $12(32)$ \\
\hline T2 & $6(16)$ \\
\hline T3 & $8(22)$ \\
\hline T4 & \\
\hline clinical N stage & $30(81)$ \\
\hline N0 & $7(19)$ \\
\hline N+ & $2(5)$ \\
\hline N stage as assessed by pathology & $26(70)$ \\
\hline after neck dissection for clinically & $9(24)$ \\
\hline N+ patients & \\
\hline N0 & $5(71)$ \\
\hline N+ & $2(29)$ \\
\hline M stage & \\
\hline M0 & \\
\hline Tumor Grading & \\
\hline G1 & \\
\hline G3 & \\
\hline
\end{tabular}

patient cohort, $\mathrm{T}$ stage was evenly distributed, with slightly less patients presenting with $\mathrm{T} 3$ tumors $(16 \%, \mathrm{n}=6)$ than $\mathrm{T} 1(27 \%$, $\mathrm{n}=10), \mathrm{T} 2(30 \%, \mathrm{n}=11)$ and T4 $(27 \%, \mathrm{n}=10)$ tumors. A detailed overview over tumor localization is provided in Supplemental Table 1. Clinically enlarged lymph nodes were observed at the time of diagnosis in 19\% ( $n=7)$ of patients, as assessed by ultrasound and contrast-enhanced CT-scan, and were therefore classified as clinically $\mathrm{N}$-positive prior to treatment.

\section{Treatment of the primary tumor}

Four out of 37 patients were treated with primary radiation therapy for the following reasons: three patients did not qualify for general anesthesia due to comorbidities and one patient refused surgical treatment. The extent of treatment performed in the remaining 33 patients is summarized in Table 3. Depending on the location and extent of the primary tumor, tumors were locally excised with adequate surgical margins, or, if adequate margins were otherwise not achievable, partial, or total rhinectomy were performed. Partial rhinectomy was performed in 8 patients, while 9 patients received a total rhinectomy and subsequent epithetic reconstruction (Figure 1). Local adjuvant radiotherapy was applied in 10 patients with either advanced
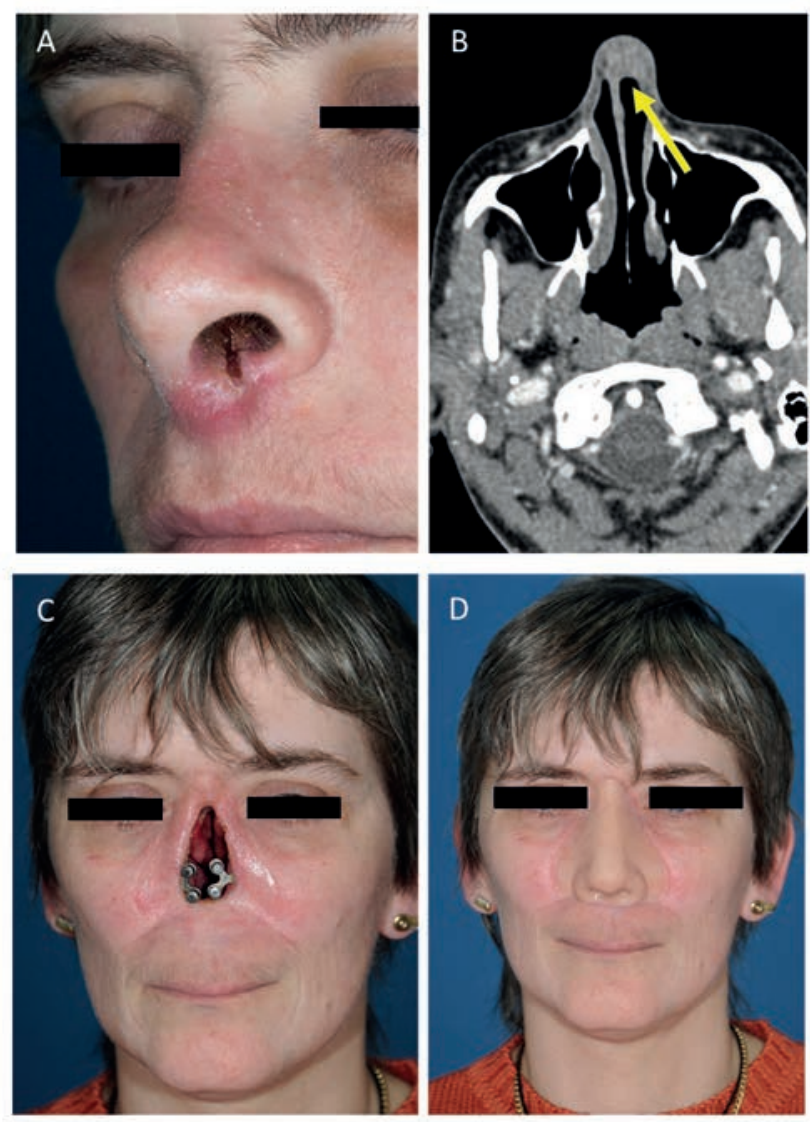

Figure 1. A. Pre-operative images of a patient with a T4 squamous cell carcinoma of the nasal cavity with infiltrating the skin. B. Pre-operative CT-scan showing the tumor originating from the nasal septum (arrow).

C. Image of the same patient after tumor resection via total rhinectomy and partial resection and reconstruction of the upper lip. Bone implants for epithetic reconstruction have been inserted. D. Image of the same patient after completion of facial reconstruction with a bone-anchored silicone nasal epithesis.

stage primary tumors (i.e. stage T4) or when tumor resection could not be performed with adequate surgical margins

Treatment of the neck

Fifteen out of 33 patients (45\%), who were treated with a primary surgical approach underwent neck dissection simultaneously with tumor resection. Seven out of 33 (21\%) of surgically treated patients presented with enlarged cervical lymph nodes at the time of diagnosis. In 2 patients, cervical lymph nodes showed signs of necrosis, as assessed by ultrasound and CT scan. In one case, lymph nodes were affected bilaterally, involving levels I through $\mathrm{V}$. The second patient showed a necrotic lymph node in level I of the neck. In 5 patients, ultrasound and CT scan revealed multiple slightly enlarged lymph nodes with a diameter of $1 \mathrm{~cm}$ to $2 \mathrm{~cm}$ which did not show signs of necrosis. Submandibular, i.e. level I nodes were affected in all patients. Neck dissection 
Table 3. Treatment Overview by Tumor Stage for 33 patients analyzed in the study which were treated with a primary surgical approach.

\begin{tabular}{|c|c|}
\hline Treatment by Tumor Stage & $\mathbf{n}=\mathbf{3 3}$ \\
\hline Tumor Stage & $\begin{array}{l}\text { No. of } \\
\text { Patients }\end{array}$ \\
\hline T1N0M0 & 10 \\
\hline Tumor Resection alone & 6 \\
\hline Tumor Resection, Neck Dissection & 2 \\
\hline T2NOMO & 9 \\
\hline Tumor Resection alone & 2 \\
\hline Tumor Resection, Neck Dissection & 5 \\
\hline Tumor Resection, Neck Dissection, Local Radiation & 2 \\
\hline $\mathrm{T} 2 \mathrm{~N} 2 \mathrm{MO}$ & 2 \\
\hline Tumor Resection, Neck Dissection & 1 \\
\hline $\begin{array}{l}\text { Tumor Resection and Neck Dissection, Local and Regi- } \\
\text { onal Radiation }\end{array}$ & 1 \\
\hline T3NOMO & 6 \\
\hline Tumor Resection alone & 2 \\
\hline Tumor Resection, Neck Dissection & 1 \\
\hline Tumor Resection, Neck Dissection, Local Radiation & 2 \\
\hline T4NOMO & 10 \\
\hline Tumor Resection alone & 2 \\
\hline Tumor Resection, Local Radiation & 2 \\
\hline Tumor Resection, Local and Regional Radiation & 3 \\
\hline Tumor Resection, Neck Dissection, Local Radiation & 2 \\
\hline
\end{tabular}

T-stage for all patients refers to pathologically confirmed T-stage. $\mathrm{N}$-stage refers to pathologically confirmed $\mathrm{N}$-stage for patients who underwent neck dissection and clinical N-stage for those who did not.

was performed in all 7 cases; here, lymph node metastases were histologically confirmed in the two cases which had shown signs of necrosis. None of the lymph node metastases showed signs of extracapsular spread. None of the patients who received neck dissection without suspicious lymph node features $(n=8)$ showed occult lymph node metastases as assessed by histology. Indication for adjuvant regional radiotherapy was based on tumor extension and the results of histological analysis. Regional radiation of the cervical lymph nodes was performed in 3 out of 9 surgically treated patients with T4 tumors. 2 patients with T4 tumors received extensive bilateral neck dissection which showed no histological indication of lymph node metastasis and therefore only underwent local irradiation of the tumor region only. Four further patients had small tumors classified as T4 due to skin infiltration and therefore did not receive any treatment of

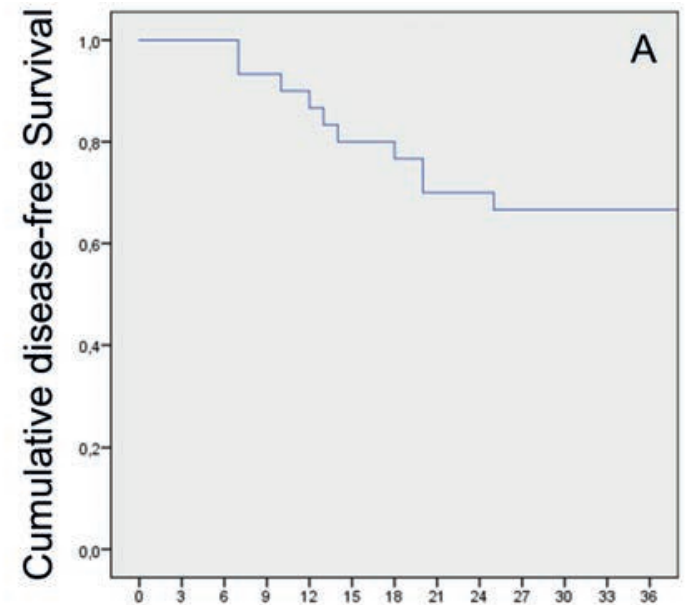

Time in Months

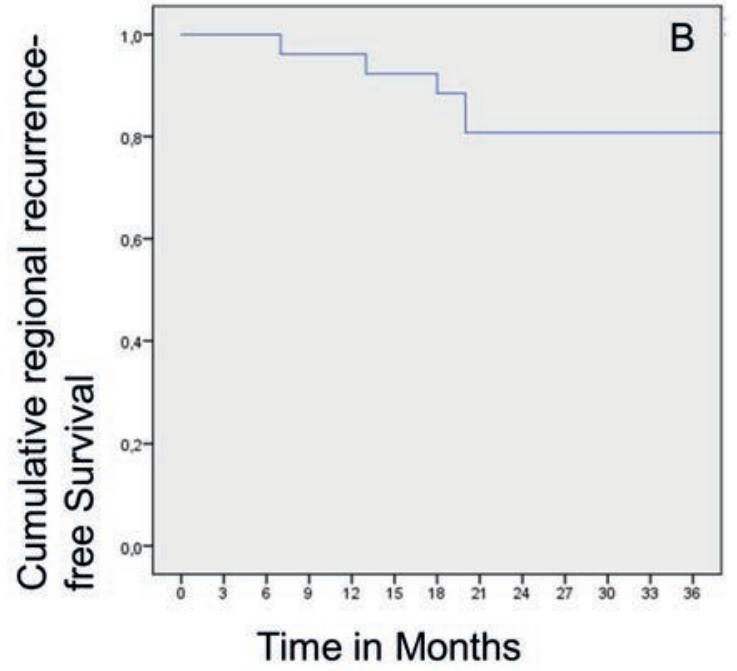

Figure 2. A, Kaplan-Meier curve of 3-year disease-free survival for all patients included in the study with at least a 3-year follow-up period $(n=30)$. Disease-free survival is shown to be $63 \%$ with the majority of recurrence occurring within the first 24 months. B. Kaplan-Meier curve for regional recurrence-free survival for surgically treated patients with at least a 3-year follow-up period ( $n=26)$. Regional recurrence-free survival is shown to be $77 \%$ with the majority of events occurring in the first 24 months.

the neck per tumor board decision.

One patient with histologically confirmed lymph node metastases received adjuvant radiotherapy of the neck, while the second patient with confirmed lymph node metastases at the time of diagnosis refused adjuvant radiotherapy.

\section{Therapeutic outcome}

Follow-up for patients in this study ranged from 10 months to 142 months. Overall disease-free survival for all patients in this study was $77 \%$. In order to allow for a sufficient follow-up period, disease-free survival for patients who had completed a follow-up period of $>3$ years $(n=30)$ at the time of the study 
Table 4. Overview over cases with cervical lymph node recurrence after primary surgical therapy.

\begin{tabular}{|c|c|c|c|}
\hline Initial Stage & Initial Treatment & $\begin{array}{l}\text { Location of Lymph Node } \\
\text { Recurrence }\end{array}$ & $\begin{array}{l}\text { Time since } \\
\text { Surgery }\end{array}$ \\
\hline T1NOM0 & local tumor resection & level I ipsilateral side & 20 months \\
\hline T2NOMO & local tumor resection, ipsilateral neck dissection & level I contralateral side & 13 months \\
\hline $\mathrm{T} 2 \mathrm{~N} 2 \mathrm{cMO}$ & tumor resection, bilateral neck dissection (patient refused adjuvant radiotherapy) & level I contralateral side & 6 months \\
\hline T3NOMO & total rhinectomy, bilateral neck dissection, local adjuvant radiotherapy & level I & 19 months \\
\hline T4NOM0 & total rhinectomy, local and regional adjuvant radiotherapy & level I & 16 months \\
\hline T4NOMO & partial rhinectomy, local and regional adjuvant radiotherapy & levels I-IV & 56 months \\
\hline
\end{tabular}

T-stage for all patients refers to pathologically confirmed T-stage. $\mathrm{N}$-stage refers to pathologically confirmed $\mathrm{N}$-stage for patients who underwent neck dissection and clinical N-stage for those who did not.

was calculated, showing a disease-free survival rate of $63 \%$. As shown in the Kaplan-Meier plot (Figure 2A), the majority of recurrences occurred within the first 24 months after initial treatment.

Two out of 4 patients treated with primary radiotherapy had a local recurrence within two years, 2 patients were disease-free. Both patients with local recurrence did not qualify for general anesthesia and therefore underwent palliative chemotherapy. Four out of 33 (12\%) patients treated with a primary surgical approach had a local recurrence. 3 of 4 patients with local recurrence had received local surgical tumor resection only, in one case with adjuvant local radiotherapy, while 1 patient had been treated with partial rhinectomy. None of the patients who had been treated with total rhinectomy had local recurrence over the course of their respective follow-up period.

Six out of all $33(18 \%)$ surgically treated patients showed cervical lymph node recurrence, 26 of these patients had at least a threeyear follow-up period (Figure 2B). A detailed overview of the treatment received by the patients with lymph node recurrence is shown in Table 4. All cervical lymph node recurrences involved level I and mostly occurred within two years after tumor surgery.

\section{Discussion}

\section{Patient collective}

Our study presents a retrospective analysis of 37 patients with NCSCC, all treated at a single institution. Compared to population-based and large multi-center analyses, the single-center approach allows for a comprehensive analysis of diagnostic and therapeutic parameters, as well as individual patient outcomes. Due to the overall rarity of this specific tumor entity, the number of patients included is in line with patient numbers reported by other single-center studies ${ }^{(7-9)}$, particularly after exclusion of cancers of the paranasal sinuses. While population-based analyses show that the majority of NCSCC patients present with early stage tumors ${ }^{(2)}$, distribution among T-stages was relatively even in our patient collective. The distribution of tumor stage can most likely be attributed to the relatively low number of patients included in this study, leading to a slight over-representation of advanced stage tumors. This needs to be taken into consideration when assessing survival data. Also, most likely due the small sample size, female patients were over-represented in our patient cohort compared to population-based studies. Outcomerelevant patient and tumor characteristics, such as age at the time of diagnosis, as well as M-stage, and importantly N-stage, were in line with those reported in larger analyses.

\section{Treatment of the primary tumor}

In the analyzed patient collective, 4 patients were treated with primary radiotherapy because a primary surgical approach was either denied or not feasible due to unrelated health concerns preventing patients from undergoing general anesthesia. The local recurrence rate for patients treated with surgery was $12 \%$. Surgery being the primary treatment option for resectable NCSCC is supported by larger studies comparing the main treatment modalities. Results reporting on recurrence rates after primary radiotherapy were around $50 \%$ and rates after surgical therapy around $20 \%{ }^{(10,11)}$. In addition, as in other single-center studies which exactly assessed the extent of surgery performed for every patient ${ }^{(9)}$, none of the patients receiving total rhinectomy showed any local recurrence in our patient cohort. These data suggest that excellent local control is achievable via complete resection of the nose. In this context, it seems important to note that while the majority of patients receiving partial or total rhinectomy had T4 tumors, 4 patients with T2 and 
T3 tumors were also treated with total rhinectomy, suggesting that T-stage alone was not the only determining factor for the extent of surgery. Here, the staging system (Table 1) needs to be kept in mind, per which even $\mathrm{T} 1$ tumors may present with bony involvement, requiring extensive resection. At the same time, even small tumors, which can be easily resected with sufficient margins may be classified as T4, if the skin is infiltrated. Particularly for tumors of the nasal vestibule, even small tumors oftentimes present with skin infiltration, mandating classification as stage T4. This may explain why in NCSCC the T-stage has less prognostic significance than in other head and neck malignancies ${ }^{(12)}$. An increase in prognostic significance of T-stage could potentially be achieved by classifying tumors of the nasal vestibule separately.

While providing excellent local control, total rhinectomy is a procedure which severely affects patients' appearance and is therefore oftentimes met with severe resistance. Hence, providing adequate facial reconstructive options is essential. In our patient cohort, all patients who underwent total rhinectomy received consequent facial reconstruction via nasal epithesis. Bone-anchored nasal epithetic reconstruction yields low complication rates and silicone prostheses provide excellent aesthetic outcomes ${ }^{(13)}$. Surgical implant anchorage can be performed simultaneously with tumor surgery, even if the patient has to undergo adjuvant therapy. While adjuvant irradiation slightly increases the rate of implant failure, implant survival rates have still been reported to be higher than $90 \%$ after irradiation ${ }^{(14)}$.

\section{Regional treatment and recurrence}

In our patient cohort, $19 \%$ of surgically treated patients presented with enlarged cervical lymph nodes at the time of diagnosis and underwent neck dissection at the time of tumor resection. Per interdisciplinary tumor board decision, neck dissection was also performed in patients with normal lymph node status who were determined to be at a higher risk for nodal involvement due to the extent of the primary tumor. In total, neck dissection was performed in $45 \%$ of surgically treated patients, revealing lymph node metastases in 2 patients, i.e. $6 \%$ of surgically treated patients. Both patients had presented with clinically enlarged, highly suspicious lymph nodes. These findings are in line with the results reported by others, showing a low rate of nodal involvement at the time of diagnosis for $\operatorname{NCSCC}{ }^{(7,9,15)}$. While these results prompted authors to suggest that elective neck dissection of the clinically negative ( $\mathrm{CNO}$ ) neck may not be warranted for NCSCC patients ${ }^{(9)}$, Scurry et al. showed in a comprehensive meta-analysis that regional recurrence rates are close to $20 \%$ across all stages for NCSCC ${ }^{(6)}$. Findings in our patient collective confirmed these data: $18 \%(n=6)$ of patients had regional recurrence over the course of the follow-up period, presenting with lymph node metastases after an average of 22 months after tu- mor resection. One patient had already presented with bilateral histologically confirmed lymph node involvement at the time of initial surgery and undergone neck dissection. Consequent adjuvant radiation was refused by the patient, and local recurrence occurred after 6 months. In the other 5 patients, regional recurrence occurred across all stages ( $1 \mathrm{~T} 1,1 \mathrm{~T} 2,1 \mathrm{~T} 3$, and $2 \mathrm{~T} 4$ tumors). None of the patients had presented with suspicious lymph nodes at the time of diagnosis. In all patients, lymph node metastases involved level I of the neck, which is part of the known lymphatic drainage pathway, which also includes facial and anterior parotid nodes ${ }^{(16)}$. To address the high rate of regional recurrence shown in their meta-analysis, Scurry et al. suggested that treatment of the neck at the time of initial surgical therapy may be warranted in patients presenting with tumor characteristics such as advanced T-stage, perineural spread, or lymphovascular invasion. Since the lymphatic drainage pathway, particularly level I of the neck, holds a high potential for surgical morbidity, adjuvant radiation instead of neck dissection was suggested. In our patient collective, regional recurrence occurred in two patients with $\mathrm{T} 1$ and $\mathrm{T} 2$ tumors without adverse tumor characteristics who both did not receive treatment of the neck, as well as in 3 patients with advanced stage tumors (1 T3, 2 T4). Importantly, both patients with T4 tumors underwent regional irradiation, while the patient presenting with a T3 tumor at the time of diagnosis underwent bilateral neck dissection. While the relatively low number of patients included in our analysis needs to be taken into consideration, our results show that $21 \%$ (3 out of 14 ) patients with advanced stage, i.e. stage T3 and T4 NCSCC, developed regional recurrence despite having undergone monomodal treatment of the neck. None of the patients showed any indication of nodal metastasis at the time of diagnosis. While larger analyses of patients with advanced stage NCSCC may be necessary to support these findings, our results suggest that patients with $\mathrm{T} 3$ and $\mathrm{T} 4$ tumors are at a high risk of regional recurrence. Importantly, regional recurrence occurred despite prior surgical treatment or irradiation of the neck. Notably, none of the patients in which regional recurrence occurred, underwent multimodal treatment of the neck. Aggressive treatment involving a combination of neck dissection, including thorough bilateral resection of cervical level I which was involved in all patients who showed regional recurrence in our study, and regional radiotherapy may need to be taken into consideration to further reduce the risk of regional recurrence. 3 patients in our cohort with T4 tumors underwent bimodal treatment of the neck and showed no signs of regional recurrence. For patients with early stage NCSCC and no evidence of lymph node metastasis at the time of diagnosis, rates of regional recurrence were substantially lower at $9 \%$ (2 of 21 patients) but nevertheless should be taken into careful consideration during therapy and follow-up. 


\section{Conclusion}

Overall, the results obtained in this study for patients across stages suggest that while NCSCC patients mostly present without clinically, or histologically positive lymph nodes at the time of diagnosis, NCSCC can by no means be considered a disease with rare lymph node involvement. Treatment regimens need to take the delayed occurrence of lymph node metastases into careful consideration. Due to the rarity of the disease, larger studies from which definitive treatment recommendations can be derived are not available. Although in a small sample size, the presented findings suggest that bimodal treatment of the neck may be necessary to effectively prevent regional recurrence, particularly for advanced stage tumors, and treating physicians should counsel patients accordingly. Clinical follow-up after primary treatment by cervical ultrasound with utmost attention being paid to cervical level I is critical for all stages and needs to be performed regularly Within the first two years, follow-up intervals should not exceed 6-8 weeks.

\section{Authorship contribution}

Conception and design:T.H. and B.A.S.

Data acquisition and analysis: T.H. and B.A.S.

Writing and review of the manuscript: all authors.

\section{Conflict of interest}

The authors declare that they have no conflict of interest.

\section{References}

1. Dulguerov $\mathrm{P}$, Jacobsen MS, Allal AS, Lehmann W, Calcaterra T (2001) Nasal and paranasal sinus carcinoma: are we making progress? A series of 220 patients and a systematic review. Cancer 92 (12):3012-3029.

2. Unsal AA, Dubal PM, Patel TD, Vazquez A, Baredes S, Liu JK, Eloy JA (2016) Squamous cell carcinoma of the nasal cavity: A population-based analysis. The Laryngoscope 126 (3):560-565. doi:10.1002/lary.25531.

3. Sanghvi S, Khan MN, Patel NR, Yeldandi S, Baredes S, Eloy JA (2014) Epidemiology of sinonasal squamous cell carcinoma: a comprehensive analysis of 4994 patients. The Laryngoscope 124 (1):76-83.

4. Redaelli de Zinis LO, Nicolai P, Tomenzoli D, Ghizzardi D, Trimarchi M, Cappiello J, Peretti G, Antonelli AR (2002) The distribution of lymph node metastases in supraglottic squamous cell carcinoma: therapeutic implications. Head \& neck 24 (10):913-920.

5. Katz TS, Mendenhall WM, Morris CG, Amdur RJ, Hinerman RW, Villaret DB (2002) Malignant tumors of the nasal cavity and paranasal sinuses. Head \& neck 24 (9):821829.

6. Scurry WC, Jr., Goldenberg D, Chee MY, Lengerich EJ, Liu Y, Fedok FG (2007) Regional recurrence of squamous cell carcinoma of the nasal cavity: a systematic review and meta-analysis. Archives of otolaryngology--head \& neck surgery 133 (8):796-800
7. Dirix P, Nuyts $S$, Geussens $Y$, Jorissen $M$ Vander Poorten V, Fossion E, Hermans R Van den Bogaert W (2007) Malignancies of the nasal cavity and paranasal sinuses: longterm outcome with conventional or threedimensional conformal radiotherapy. Int J Radiat Oncol Biol Phys 69 (4):1042-1050.

8. Guan X, Wang X, Liu Y, Hu C, Zhu G (2013) Lymph node metastasis in sinonasal squamous cell carcinoma treated with IMRT/3DCRT. Oral oncology 49 (1):60-65.

9. Becker C, Kayser G, Pfeiffer J. Squamous cell cancer of the nasal cavity: New insights and implications for diagnosis and treatment. Head Neck. 2016 Apr;38 Suppl 1:E2112-7.

10. Mendenhall WM, Amdur RJ, Morris CG, Kirwan J, Malyapa RS, Vaysberg M, Werning JW, Mendenhall NP (2009) Carcinoma of the nasal cavity and paranasal sinuses. The Laryngoscope 119 (5):899-906.

11. Turner JH, Reh DD (2012) Incidence and survival in patients with sinonasal cancer: a historical analysis of population-based data. Head \& neck 34 (6):877-885.

12. Bhattacharyya $N$ (2002) Cancer of the nasal cavity: survival and factors influencing prognosis. Archives of otolaryngology-head \& neck surgery 128 (9):1079-1083.

13. Papaspyrou G, Schick B, Schneider M, A Kadah B. Epithetic nasal reconstruction for nasal carcinoma: retrospective analysis on 22 patients. Eur Arch Otorhinolaryngol. 2017 Feb;274(2):867-872.

14. Schoen PJ, Raghoebar GM, van Oort RP,
Reintsema H, van der Laan BF, Burlage FR, Roodenburg JL, Vissink A (2001) Treatment outcome of bone-anchored craniofacial prostheses after tumor surgery. Cancer 92 (12):3045-3050.

15. Fornelli RA, Fedok FG, Wilson EP, Rodman SM. Squamous cell carcinoma of the anterior nasal cavity: a dual institution review. Otolaryngol Head Neck Surg. 2000 Sep;123(3):207-10.

16. Day TA, Beas RA, Schlosser RJ, Woodworth BA, Barredo J, Sharma AK, Gillespie MB (2005) Management of paranasal sinus malignancy. Curr Treat Options Oncol 6 (1):3-18.

Timon Hussain, MD

Department of Otorhinolaryngology

Head and Neck Surgery

Essen University Hospital

Hufelandstr. 55

45147 Essen

Germany

Tel: +49 (0)201723 2481

E-mail: timon.hussain@uk-essen.de 
Supplemental Table 1. Detailed overview over tumor localization for all patients included in the study.

\begin{tabular}{|c|c|c|}
\hline $\begin{array}{l}\text { Patient } \\
\text { no. }\end{array}$ & T-Stage & Tumor localization by subsites \\
\hline 1 & T1 & lateral wall \\
\hline 2 & T1 & vestibule \\
\hline 3 & T1 & vestibule \\
\hline 4 & T1 & septum \\
\hline 5 & $\mathrm{~T} 1$ & vestibule \\
\hline 6 & T1 & vestibule \\
\hline 7 & T1 & lateral wall \\
\hline 8 & T1 & lateral wall \\
\hline 9 & T1 & vestibule \\
\hline 10 & T1 & vestibule \\
\hline 11 & T1 & lateral wall \\
\hline 12 & $\mathrm{~T} 2$ & lateral wall, septum \\
\hline 13 & $\mathrm{~T} 2$ & lateral wall, septum \\
\hline 14 & $\mathrm{~T} 2$ & lateral wall, septum \\
\hline 15 & $\mathrm{~T} 2$ & vestibule, septum \\
\hline 16 & $\mathrm{~T} 2$ & vestibule, lateral wall \\
\hline 17 & $\mathrm{~T} 2$ & lateral wall, septum \\
\hline 18 & $\mathrm{~T} 2$ & lateral wall, septum \\
\hline 19 & $\mathrm{~T} 2$ & lateral wall, septum \\
\hline 20 & $\mathrm{~T} 2$ & lateral wall, septum \\
\hline 21 & $\mathrm{~T} 2$ & vestibule, septum \\
\hline 22 & $\mathrm{~T} 2$ & lateral wall, septum \\
\hline 23 & $\mathrm{~T} 2$ & lateral wall, septum \\
\hline 24 & T3 & septum with bony palate invasion \\
\hline 25 & T3 & lateral wall, septum, bony palate invasion \\
\hline 26 & T3 & lateral wall, septum, bony palate invasion \\
\hline 27 & T3 & septum, bony palate invasion \\
\hline 28 & T3 & lateral wall, septum, bony palate invasion \\
\hline 29 & T3 & lateral wall, septum, bony palate invasion \\
\hline 30 & T4 & septum, vestibule with skin infiltration \\
\hline 31 & T4 & septum, vestibule with skin infiltration \\
\hline 32 & T4 & vestibule with skin invasion \\
\hline 33 & T4 & septum, vestibule with skin infiltration \\
\hline 34 & T4 & $\begin{array}{l}\text { septum, lateral wall, nasal vestibule with skin } \\
\text { infiltration }\end{array}$ \\
\hline 35 & T4 & septum, vestibule with skin infiltration \\
\hline 36 & T4 & $\begin{array}{l}\text { septum, lateral wall, vestibule with skin infiltra- } \\
\text { tion }\end{array}$ \\
\hline 37 & T4 & lateral wall, septum with skin infiltration \\
\hline
\end{tabular}

